\title{
Health-related quality of life in Hodgkin lymphoma: a systematic review
}

\author{
Nadine Linendoll ${ }^{1,4^{*}}$, Tully Saunders ${ }^{2}$, Rebecca Burns ${ }^{2}$, Jonathan D. Nyce ${ }^{4}$, Kristen B. Wendell ${ }^{3}$ \\ Andrew M. Evens ${ }^{1,4}$ and Susan K. Parsons $s^{1,2,4}$
}

\begin{abstract}
Purpose: Hodgkin Lymphoma $(\mathrm{HL})$ is highly curable with well-established treatment regimens; however, the impact on patient's health-related quality of life (HRQL) from diagnosis through survivorship is unclear. This systematic review aimed to describe the available literature on $\mathrm{HRQL}$ in $\mathrm{HL}$, assess the quality of these studies, identify gaps in the literature and recommend further areas of research.

Methods: Following PRISMA guidelines, we performed a systematic review to include studies assessing the HRQL in $\mathrm{HL}$ patients. Articles identified through database searches were screened and data extracted. Quality was evaluated using a 6-point scale, adapted from published HRQL systematic reviews.

Results: Sixty five articles published between 1986 and 2015 met inclusion criteria. These included 53 (82 \%) crosssectional studies; 12 (18\%) longitudinal studies, including three embedded in randomized trials; and three additional longitudinal studies that began assessment at diagnosis. Study sample sizes of HL patients varied considerably with only five (42\%) longitudinal studies including more than 50 patients. Multidimensional HRQL was assessed in 45 studies, single HRQL domains in 22 studies, and symptoms, including fatigue, in 28 studies.

Conclusions: The majority of studies employed a cross-sectional design, enrolling HL survivors at least 10 years after the completion of therapy. Emphasis on HRQL following therapy may inform initial treatment decisions and long-term survivorship goals. We recommend that future research include prospective, longitudinal randomized designs across both treatment and time.
\end{abstract}

Keywords: Hodgkin lymphoma, Quality of life, Survivorship, Systematic review

\section{Background}

Hodgkin Lymphoma (HL) has well established treatment regimens that have yielded highly effective, long-term cure rates [1,2]. In 2015, there were an estimated 9050 new cases of the disease, which is a much lower incidence than more commonly occurring cancers. The number of persons living with HL in 2012, however, was estimated to approach 190,000 [3]. Thus, from an oncologic perspective the successful treatment for $\mathrm{HL}$ over the last 25 years has led to a high number of long-term survivors.

\footnotetext{
* Correspondence: nlinendoll@tuftsmedicalcenter.org

${ }^{1}$ Tufts Cancer Center, Tufts Medical Center, 800 Washington Street, \#245, Boston, MA 02111, USA

${ }^{4}$ Tufts University School of Medicine, 145 Harrison Avenue, Boston, MA 02111, USA

Full list of author information is available at the end of the article
}

The incidence of $\mathrm{HL}$ is bimodal by age with the first peak within adolescence and young adulthood, ages 15-40, and the second after age 55 [4]. For younger people with HL, the aggressive cancer treatment often interrupts important developmental milestones, such as graduation from high school or college, establishing relationships, finding a first apartment or getting a job. Despite the decades of curative therapy for HL, comparatively little is known about how $\mathrm{HL}$ affects health-related quality of life (HRQL) - through diagnosis and treatment. Therefore, healthcare providers lack the information that they need regarding how best to intervene in a timely and effective manner to mediate HL's acute and late-term effects.

HRQL is a multidimensional construct, which reflects the World Health Organization's definition of health as incorporating physical, mental, and social health [5]. The history of $\mathrm{HRQL}$ assessment largely parallels the advances 
in HL therapy with most of the instrument development and large-scale validation studies for adults commencing in the 1980's. The first randomized controlled trial (RCT) containing an HRQL outcome was reported in 1986 by Croog and Levine [6]. In contrast, instrument development for children lagged considerably, but psychometrically robust measures are now available for most patients across the age continuum. Several validated instruments address the multidimensional construct, while others focus on individual dimensions or domains of HRQL, such as physical or psychosocial functioning. Still others measure individual symptoms, such as pain, nausea or fatigue. The selection of a particular instrument or instruments is informed by the particular study question.

Although treatment regimens are well-established, the impact on HL patient's HRQL from diagnosis through long-term survivorship is still unclear. In this study, we report on a systematic review that we conducted on the HRQL literature to answer the following research question: What is the HRQL impact of HL and its treatment as reflected in the current literature? Our goals were to: (1) describe the available literature on HRQL in HL, (2) assess the quality of these studies and, (3) identify gaps in the available literature and recommend further areas of research.

\section{Methods}

\section{Literature search strategy}

Our review was guided by the PRISMA statement [7]. We searched Medline, CINAHL and PsychInfo using Medical Subject Headings and keywords, such as Hodgkin disease, quality of life, health-related quality of life, well-being, functional status, health status and experiential health status for articles published since inception to end of May 2016 that reported primary HRQL data specific to HL patients. A priori, we sought studies that assessed single or multi-dimensional HRQL domains or discrete symptoms, such as fatigue. Study design was not part of the inclusion/exclusion criteria as our goal was to examine the entirety of the HRQL in HL literature. Studies were excluded if they (1) did not report primary HRQL separately for HL patients or (2) were not available in English. Following this database search, a citation analysis was performed on review articles to identify additional articles (see Fig. 1). This was done to ensure that this review afforded the most comprehensive representation of the literature. Selected articles included HL patients across the age (children and adults) and care continuum, from initial diagnosis through long-term survivorship.

\section{Review of the abstracts and full-text articles}

The six-member team initially reviewed a training set of 50 abstracts for consideration in the study, applying established inclusion/exclusion criteria to ensure consistency across all team members. Subsequently, teams of two reviewers independently applied inclusion/exclusion criteria to the remaining abstracts and then full text articles, resolving any discrepancies through consensus. Full text articles meeting inclusion criteria were independently data extracted using a standardized data extraction form and checked for accuracy by a second review.

Review articles were handled differently from original reports. Because the review articles did not include original data, we did not perform data extraction on review articles. The review articles provided background information and were used in the citation analysis.

\section{Quality indicators}

The methodological quality of each study was evaluated by a set of six predetermined criteria, adapted from previously published systematic reviews $[8,9]$. Quality assessment (QA) criteria included: (1) description of $\geq 2$ demographic variables specific to HL patients, such as age and gender; $(2) \geq 2$ clinical characteristics, specific to HL patients, such as stage or site of disease; (3) sample size $\geq 50$ HL participants; (4) HL-specific HRQL scores; presented as a mean summary score and measure of variability in either table or text format; (5) analysis of HRQL scores by HL specific demographic characteristics and (6) HL results compared within groups. The final QA checklist scores were summed by each article to give an overall quality score, ranging from 0 to 6 in which higher scores indicated higher quality.

\section{Results}

The database search totaled 792 unique records. Following the screening process, 80 studies, published between 1986 and 2015, were identified, including 65 primary studies and 15 review articles. Reasons for rejection of full text articles included HL data not separated out, no HRQL data reported, not available in English, or an editorial article. The following section reports on the 65 primary studies.

\section{Study characteristics}

Study characteristics are presented in Table 1. Of the 65 studies, 53 (82\%) utilized cross-sectional design; only 12 (18\%) used longitudinal design. Of the 12 longitudinal studies, seven included individuals who were off treatment, while five included samples who were both on and off treatment. We identified three longitudinal RCTs [1012], of which patients were followed from the time of diagnosis through up to 10 years of survivorship. These studies accrued large samples and made comparison of HRQL according to randomized treatment groups. The 53 cross sectional studies reported on 35 unique cohorts and only one included patients both on and off treatment. 


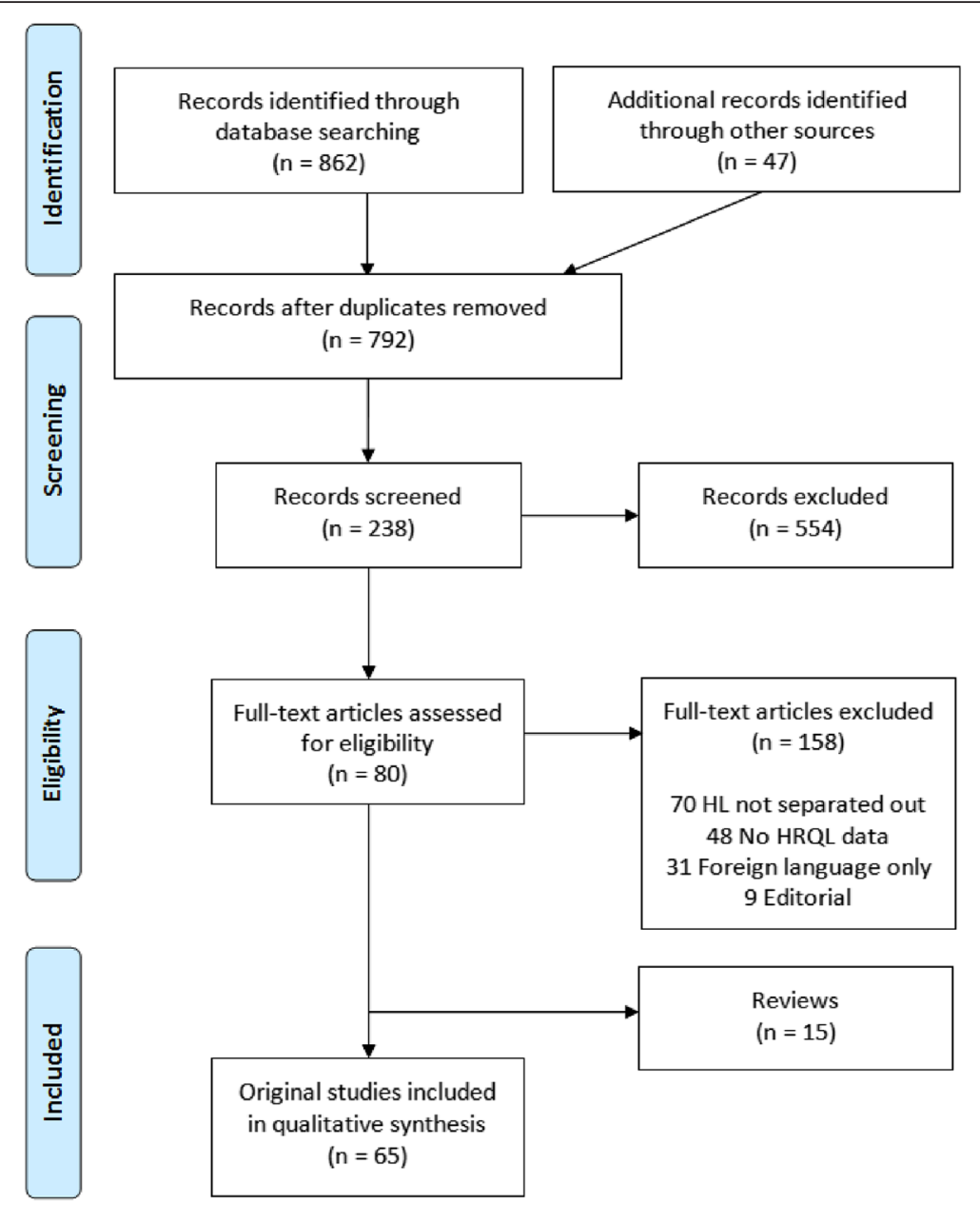

Fig. 1 Systematic review flow diagram following PRISMA guidelines

The remaining 52 (98\%) cross-sectional studies only included individuals who were off active treatment.

The sample size of HL patients varied considerably with $78 \%$ of studies including a sample of 50 or more HL patients. For the 53 cross-sectional studies, the median sample size was 135 (range 15-1843) and for the 12 longitudinal studies the median sample size was 51 (range 12-3208). The majority of study designs were cohort $(n=45)$ and 14 used a comparator cohort design. The most common comparators were the general population $(n=23)$, other cancer patients $(n=12)$ and siblings $(n=3)$. When comparing among other cancer patients, the most common comparator included other types of lymphoma $(n=7)$. The majority of studies relied on remote data collection $(n=42)$, when specified either by mail $(n=35)$ or telephone $(n=6)$, with the remainder conducted in person. The study funding was primarily investigator initiated $(n=58)$ or via a cooperative group $(n=7)$. We did not identify any industry-funded studies. Half (52\%) of the identified studies were published after 2005. Table 2 contains the data extraction results.

\section{Patient characteristics}

Age was reported in 54 studies (83\%). Thirty-three studies reported a mean age of 40 years $(\mathrm{SD}=8.5)$ and 21 studies reported median age of 38 years (range 22-52). In 41 (63\%) studies that reported time since diagnosis, the mean was 11.8 years $(n=25)$ and the median was 12.4 years $(n=$ 16). Twelve (19\%) studies reported time post treatment, with a mean of 4.8 years $(\mathrm{SD}=1.73)(n=4)$ and a median of 10.2 years $(n=3)$. Five studies reported only a range of years post treatment (1-23 years).

\section{Quality of life measures}

Studies varied by aspect of HRQL examined as well as by specific instrument used. Table 3 summarizes the various aspects of HRQL by study design (longitudinal vs. cross-sectional). The most commonly used multidimensional instruments were the EORTC QLQ-C30 $(n=18)$, the SF-12 or SF-36 (SF-36 Family) $(n=18)$ and Health Utilities Index (HUI) 2, $3(n=8)$. Strikingly, all 12 longitudinal studies included a multidimensional scale whereas only $62 \%(n=33)$ of the cross-sectional 
Table 1 Study characteristics $(N=65)$

\begin{tabular}{|c|c|}
\hline \multicolumn{2}{|l|}{ Study design, $n$ (\%) } \\
\hline Comparator: RCT & $3(5)$ \\
\hline Comparator: cohort & $14(22)$ \\
\hline Cohort & $45(69)$ \\
\hline Comparator: case control & $3(5)$ \\
\hline \multicolumn{2}{|l|}{ Study type, $n(\%)$} \\
\hline Longitudinal & $12(18)$ \\
\hline Cross-sectional & $53(82)$ \\
\hline \multicolumn{2}{|l|}{ Study setting, $n$ (\%) } \\
\hline In person & $18(28)$ \\
\hline Remote & $42(65)$ \\
\hline NS & $5(8)$ \\
\hline \multicolumn{2}{|l|}{ Decade of publication } \\
\hline Before 1995 & $6(9)$ \\
\hline 1995 to 2004 & $25(38)$ \\
\hline 2005 to June 2015 & $34(52)$ \\
\hline \multicolumn{2}{|l|}{ Study sponsor, n (\%) } \\
\hline Cooperative group & $7(11)$ \\
\hline Investigator initiated & $58(89)$ \\
\hline \multicolumn{2}{|l|}{ Geographic location, n (\%) } \\
\hline USA & $19(29)$ \\
\hline Norway & $17(26)$ \\
\hline Netherlands & $7(11)$ \\
\hline Germany & $4(6)$ \\
\hline Other Europe & $10(15)$ \\
\hline South America & $4(6)$ \\
\hline Canada & $3(5)$ \\
\hline Asia & $1(2)$ \\
\hline \multicolumn{2}{|l|}{ n (HL patients) } \\
\hline Median (range) & $132(12-3208)$ \\
\hline $25-75^{\text {th }}$ percentile & $58-280$ \\
\hline \multicolumn{2}{|l|}{ Treatment status, $n$ (\%) } \\
\hline Active and off treatment & $6(9)$ \\
\hline Off treatment only & $59(91)$ \\
\hline \multicolumn{2}{|l|}{$\mathrm{Age}^{\mathrm{a}}$} \\
\hline Mean $(n=33)$ & 39.6 \\
\hline Median $(n=21)$ & 38 \\
\hline \multicolumn{2}{|l|}{ Gender, \% } \\
\hline Female $(n=54)$ & $44.0 \%$ \\
\hline Range & $0-100 \%$ \\
\hline
\end{tabular}

${ }^{a}$ Age: 3 studies with multiple arms, 6 studies NS $R C T$ randomized controlled trial, NS not stated

studies did so. Among cross-sectional studies, the most commonly used measures were the HADS $(n=8)$, Fatigue Questionnaire $(n=7)$, and the EORTC QLQ-C30 $(n=7)$.
Multi-dimensional HRQL scores varied by treatment type [10, 13, 14], age and sex [11], comorbidities [15] or late effects [16-18]. Both Khimani and Adams found significantly lower quality of life among HD survivors who experienced cardiopulmonary late effects [16, 18]. Goodman and Minn found that HD patients undergoing autologous stem cell transplant reported no difference in global HRQL versus the general population, however these patients did experience a decrease in overall cognitive and social health $[19,20]$. Finally, Ganz found that HRQL can improve over the trajectory of treatment into survivorship [10] and Mols supported this finding that HRQL continues to improve through long-term survivorship [21].

Among the single domains explored, psychosocial was the most frequently identified with the psychosocial assessments more common in cross-sectional (38 \%) compared to longitudinal (33\%) studies. The most frequently used psychosocial instruments included the Hospital Anxiety and Depression Scale (HADS) $(n=13)$, the Brief Symptom Inventory Scale (BSI) $(n=6)$, the Profile of Mood States (POMS) $(n=5)$ and the Psychosocial Adjustment to Illness Scale (PAIS) $(n=5)$. As studies utilized a variety of scales and measures, the results within this domain were inconsistent and the presence of psychosocial distress varied among reports. Early studies indicated that HL survivors experienced increased psychological distress [22-24], which was later supported by Oerlemans [25]; however, several others studies found no difference in the psychosocial distress of HL survivors when compared to healthy controls [26-28].

The second most commonly assessed domain was sexual health $(n=7)$. When assessing sexual health, researchers used four validated sexual health instruments including the Sexual Health Scale, the Global Sexual Satisfaction Index, the Brief Sexual Function Inventory, and the Sexual Function Scale. Four of the studies indicated that HL survivors report increased SX problems [14, 22, 29, 30]. These SX problems can improve over time [11], but may be more long lasting in higher-stage patients [12].

Several of studies used symptom-specific questionnaires $(n=29)$. Of these symptom assessments, the $76 \%$ majority focused on fatigue $(n=22)$. Other symptom assessments included nausea $(n=3)$, energy level $(n=1)$, pain $(n=1)$ and symptom distress $(n=1)$. The three most commonly used fatigue instruments included the Fatigue Questionnaire (FQ) $(n=12)$, the MFI $(n=5)$, and the Functional Assessment of Chronic Illness Therapy-Fatigue (FACIT-Fatigue) $(n=3)$. Results in this domain were more homogenous with multiple studies indicating that HL survivors are at increased risk for fatigue when compared to healthy controls [10, 31-34]. Studies within this domain found correlations between fatigue and depression ([15, $32]$ and fatigue and cardiopulmonary late effects [18, 35]. 
Table 2 Studies assessing HRQL in Hodgkin lymphoma patients $(N=65)$

\begin{tabular}{|c|c|c|c|c|c|c|c|c|c|c|}
\hline Primary Author & Year & Design & Study Type & $\begin{array}{l}\text { Timepoints } \\
\text { (as reported) }\end{array}$ & $\begin{array}{l}\mathrm{N} \text { (HL } \\
\text { Specific) }\end{array}$ & $\begin{array}{l}\text { Age } \\
\text { (HL specific) }\end{array}$ & $\begin{array}{l}\text { Tx } \\
\text { Status }\end{array}$ & Measures & $\begin{array}{l}\text { Domains } \\
\text { Assessed }\end{array}$ & $\begin{array}{l}\text { Quality } \\
\text { Score }\end{array}$ \\
\hline Fobair P [26] & 1986 & Cohort & $\begin{array}{l}\text { Cross- } \\
\text { sectional }\end{array}$ & $\begin{array}{l}\text { Median } 9 \text { years post } \\
\text { treatment }\end{array}$ & 403 & 36.3 mean & Off & $\begin{array}{l}\text { CES-D, Study-specific } \\
\text { questionnaire }\end{array}$ & $\begin{array}{l}\text { Domain, } \\
\text { Symptom }\end{array}$ & 6 \\
\hline Kornblith AB [22] & 1990 & Cohort & $\begin{array}{l}\text { Cross- } \\
\text { sectional }\end{array}$ & $\begin{array}{l}6.3 \text { years mean post } \\
\text { treatment }\end{array}$ & 273 & 37 mean & Off & $\begin{array}{l}\text { PAIS-SR, BSI, POMS, IES, Global } \\
\text { Sexual Satisfaction Index }\end{array}$ & Domain & 5 \\
\hline Chao NJ [37] & 1992 & Cohort & Longitudinal & $<1$ year post treatment & 24 & $N / S$ & Off & $\begin{array}{l}\text { Study-specific } \\
\text { questionnaire }\end{array}$ & MultiD & 2 \\
\hline Kornblith AB [23] & 1992 & Cohort & $\begin{array}{l}\text { Cross- } \\
\text { sectional }\end{array}$ & $\begin{array}{l}\text { Mean } 6.3 \text { years post } \\
\text { treatment }\end{array}$ & 273 & 37 mean & Off & $\begin{array}{l}\text { PAIS-SR, BSI, POMS, Global } \\
\text { Sexual Satisfaction Index }\end{array}$ & Domain & 6 \\
\hline Kornblith [48] AB & 1992 & Cohort & $\begin{array}{l}\text { Cross- } \\
\text { sectional }\end{array}$ & $\begin{array}{l}\text { Mean } 2.2 \text { years post } \\
\text { treatment }\end{array}$ & 93 & 35 median & Off & PAIS-SR, BSI, POMS, IES & Domain & 4 \\
\hline Bloom JR [49] & 1993 & $\begin{array}{l}\text { Comparator: } \\
\text { Cohort }\end{array}$ & $\begin{array}{l}\text { Cross- }^{-} \\
\text {sectional }\end{array}$ & $\begin{array}{l}1 \text { or more years post } \\
\text { treatment }\end{array}$ & 85 & 32.3 mean & Off & $\begin{array}{l}\text { POMS, CES-D, Social } \\
\text { Activity Scale }\end{array}$ & Domain & 6 \\
\hline van Tulder MW [30] & 1994 & $\begin{array}{l}\text { Comparator: } \\
\text { Cohort }\end{array}$ & $\begin{array}{l}\text { Cross- } \\
\text { sectional }\end{array}$ & $\begin{array}{l}\text { Mean } 14 \text { years since } \\
\text { diagnosis }\end{array}$ & 81 & 43.6 mean & Off & $\begin{array}{l}\text { SF-36, Maudsley Martital } \\
\text { Questionnaire }\end{array}$ & $\begin{array}{l}\text { MultiD, } \\
\text { Other }\end{array}$ & 6 \\
\hline Joly F [38] & 1996 & $\begin{array}{l}\text { Comparator: } \\
\text { Case Control }\end{array}$ & $\begin{array}{l}\text { Cross- } \\
\text { sectional }\end{array}$ & $\begin{array}{l}\text { Mean } 10 \text { years since } \\
\text { diagnosis }\end{array}$ & 93 & 42 mean & Off & $\begin{array}{l}\text { EORTC QLQ-C30, Study- } \\
\text { specific questionnaire }\end{array}$ & $\begin{array}{l}\text { MultiD, } \\
\text { Other }\end{array}$ & 6 \\
\hline Norum J [50] & 1996 & Cohort & $\begin{array}{l}\text { Cross- } \\
\text { sectional }\end{array}$ & $\begin{array}{l}\text { Median } 48 \text { months since } \\
\text { diagnosis }\end{array}$ & 42 & 38 median & Off & IES, VAS & $\begin{array}{l}\text { Domain, } \\
\text { Other }\end{array}$ & 4 \\
\hline Norum J [51] & 1996 & Cohort & $\begin{array}{l}\text { Cross- } \\
\text { sectional }\end{array}$ & $\begin{array}{l}\text { Median } 48 \text { months since } \\
\text { diagnosis }\end{array}$ & 42 & 38 median & Off & EuroQol & MultiD & 4 \\
\hline Norum J [52] & 1996 & Cohort & $\begin{array}{l}\text { Cross- } \\
\text { sectional }\end{array}$ & $\begin{array}{l}\text { Median } 48 \text { months since } \\
\text { diagnosis }\end{array}$ & 42 & 38 median & Off & EORTC QLQ-C30 & MultiD & 3 \\
\hline Abrahamsen AF [29] & 1998 & Cohort & $\begin{array}{l}\text { Cross- } \\
\text { sectional }\end{array}$ & $\begin{array}{l}\text { Mean } 12 \text { years since } \\
\text { diagnosis }\end{array}$ & 459 & 44 median & Off & $\begin{array}{l}\text { Study-specific } \\
\text { questionnaire }\end{array}$ & Domain & 6 \\
\hline Kornblith AB [24] & 1998 & $\begin{array}{l}\text { Comparator: } \\
\text { Cohort }\end{array}$ & $\begin{array}{l}\text { Cross- } \\
\text { sectional }\end{array}$ & $\begin{array}{l}\text { Mean } 5.9 \text { years post } \\
\text { treatment }\end{array}$ & 273 & 37 median & Off & PAIS-SR, BSI, POMS, IES & Domain & 6 \\
\hline Greil R [13] & 1999 & Cohort & $\begin{array}{l}\text { Cross- } \\
\text { sectional }\end{array}$ & $\begin{array}{l}\text { Mean } 10.5 \text { years since } \\
\text { diagnosis }\end{array}$ & 126 & 36.9 mean & Off & EORTC QLQ-C30 & MultiD & 6 \\
\hline Kaasa S [53] & 1999 & $\begin{array}{l}\text { Comparator: } \\
\text { Cohort }\end{array}$ & $\begin{array}{l}\text { Cross- } \\
\text { sectional }\end{array}$ & $\begin{array}{l}3-20 \text { years post } \\
\text { treatment }\end{array}$ & 459 & 44 mean & Off & SF-36, FQ & $\begin{array}{l}\text { MultiD, } \\
\text { Symptoms }\end{array}$ & 5 \\
\hline Loge JH [54] & 1999 & Cohort & $\begin{array}{l}\text { Cross- } \\
\text { sectional }\end{array}$ & $\begin{array}{l}\text { Mean } 12.2 \text { years since } \\
\text { diagnosis }\end{array}$ & 459 & 44 mean & Off & HADS, FQ & $\begin{array}{l}\text { Domain, } \\
\text { Symptom }\end{array}$ & 6 \\
\hline Loge JH [55] & 1999 & Cohort & $\begin{array}{l}\text { Cross- } \\
\text { sectional }\end{array}$ & $\begin{array}{l}\text { Mean } 12.2 \text { years since } \\
\text { diagnosis }\end{array}$ & 459 & 44 mean & Off & SF-36 & MultiD & 6 \\
\hline Van Schaik CS [56] & 1999 & Cohort & $\begin{array}{l}\text { Cross- } \\
\text { sectional }\end{array}$ & $\begin{array}{l}\text { Median } 21.9 \text { years since } \\
\text { diagnosis }\end{array}$ & 33 & 21.9 median & Off & HUI2, HUI3 & MultiD & 3 \\
\hline Loge JH [32] & 2000 & Cohort & $\begin{array}{l}\text { Cross- } \\
\text { sectional }\end{array}$ & $3-23$ years post treatment & 421 & $19-74$ years & Off & $\begin{array}{l}\text { FQ, HADS, Study-specific } \\
\text { questionnaire }\end{array}$ & $\begin{array}{l}\text { Domain. } \\
\text { Symptom }\end{array}$ & 6 \\
\hline Barr RD [57] & 2001 & & & & 19 & $\mathrm{~N} / \mathrm{S}$ & Off & $\mathrm{HUI}$ & MultiD & 2 \\
\hline
\end{tabular}


Table 2 Studies assessing HRQL in Hodgkin lymphoma patients $(N=65)$ (Continued)

\begin{tabular}{|c|c|c|c|c|c|c|c|c|c|c|}
\hline & & $\begin{array}{l}\text { Comparator: } \\
\text { Cohort }\end{array}$ & $\begin{array}{l}\text { Cross- } \\
\text { sectional }\end{array}$ & $\begin{array}{l}\text { Completed treatment } \\
\text { at least } 2 \text { years prior }\end{array}$ & & & & & & \\
\hline Cameron CL [58] & 2001 & Cohort & $\begin{array}{l}\text { Cross- } \\
\text { sectional }\end{array}$ & $\begin{array}{l}\text { Median } 7 \text { years } \\
\text { since diagnosis }\end{array}$ & 272 & 37 mean & Off & $\begin{array}{l}\text { PAIS-SR, } \\
\text { Symptom Report }\end{array}$ & $\begin{array}{l}\text { Domain, } \\
\text { Symptom }\end{array}$ & 4 \\
\hline Knobel H [35] & 2001 & Cohort & $\begin{array}{l}\text { Cross- } \\
\text { sectional }\end{array}$ & $\begin{array}{l}\text { Mean } 9 \text { years } \\
\text { since diagnosis }\end{array}$ & 92 & 37 mean & Off & $\mathrm{FQ}$ & Symptom & 6 \\
\hline Zabora J [59] & 2001 & Cohort & $\begin{array}{l}\text { Cross- } \\
\text { sectional }\end{array}$ & $\begin{array}{l}58 \% \text { diagnosed } \\
\text { within } 90 \text { days } \\
\text { from study }\end{array}$ & 135 & $N / S$ & Both & BSI & Domain & 3 \\
\hline Zebrack BJ [43] & 2002 & $\begin{array}{l}\text { Comparator: } \\
\text { Cohort }\end{array}$ & $\begin{array}{l}\text { Cross- } \\
\text { sectional }\end{array}$ & $\begin{array}{l}\text { Mean } 16.2 \text { years } \\
\text { since diagnosis }\end{array}$ & 1843 & 30.8 mean & Off & BSI & Domain & 5 \\
\hline Ganz PA [10] & 2003 & $\begin{array}{l}\text { Comparator: } \\
\text { RCT }\end{array}$ & Longitudinal & $\begin{array}{l}\text { Trial registration } \\
\text { (since diagnosis) }\end{array}$ & 244 & $\begin{array}{l}\text { 31.4 STLI, } \\
33.7 \mathrm{CMT} \\
\text { median }\end{array}$ & Both & SF-36, CARES-SF, SDS & $\begin{array}{l}\text { MultiD, } \\
\text { Symptom }\end{array}$ & 4 \\
\hline Gil-Fernández JJ [27] & 2003 & Cohort & $\begin{array}{l}\text { Cross- } \\
\text { sectional }\end{array}$ & $\begin{array}{l}\text { Median } 7.6 \text { years } \\
\text { since diagnosis }\end{array}$ & 46 & 43 mean & Off & EORTC QLQ-C30, HADS & $\begin{array}{l}\text { MultiD, } \\
\text { Other }\end{array}$ & 5 \\
\hline Oldervoll LM [36] & 2003 & Cohort & Longitudinal & $\begin{array}{l}\text { Mean post treatment: } \\
6.6 \text { years fatigued } \\
\text { patients; } 4.9 \text { years } \\
\text { non-fatigued patients }\end{array}$ & 53 & $\begin{array}{l}41 \text { fatigue, } 40 \\
\text { non-fatigued } \\
\text { mean }\end{array}$ & Off & SF-36, FQ & $\begin{array}{l}\text { MultiD, } \\
\text { Symptom }\end{array}$ & 5 \\
\hline Rüffer JU [33] & 2003 & $\begin{array}{l}\text { Comparator: } \\
\text { Case Control }\end{array}$ & $\begin{array}{l}\text { Cross- } \\
\text { sectional }\end{array}$ & $\begin{array}{l}\text { Median } 5.2 \text { years } \\
\text { since diagnosis }\end{array}$ & 818 & 31 median & Off & $\begin{array}{l}\text { EORTC QLQ-C30, MFI, Life } \\
\text { Situation Questionnaire }\end{array}$ & $\begin{array}{l}\text { MultiD, } \\
\text { Symptom }\end{array}$ & 5 \\
\hline Wettergren L [60] & 2003 & Cohort & $\begin{array}{l}\text { Cross- } \\
\text { sectional }\end{array}$ & $\begin{array}{l}\text { Mean } 14 \text { years } \\
\text { since diagnosis }\end{array}$ & 121 & 47 mean & Off & SEIQol-DW & MultiD & 5 \\
\hline Adams MJ [16] & 2004 & Cohort & $\begin{array}{l}\text { Cross- } \\
\text { sectional }\end{array}$ & $\begin{array}{l}\text { Median } 14.3 \text { years } \\
\text { since diagnosis }\end{array}$ & 48 & 31.9 median & Off & $\begin{array}{l}\text { SF-36, Study-specific } \\
\text { questionnaire }\end{array}$ & MultiD & 4 \\
\hline Wettergren L [28] & 2004 & Cohort & $\begin{array}{l}\text { Cross- } \\
\text { sectional }\end{array}$ & $\begin{array}{l}\text { Mean } 13 \text { years } \\
\text { since diagnosis }\end{array}$ & 121 & 47 median & Off & SF-12, SEIQoL-DW, HADS & $\begin{array}{l}\text { MultiD, } \\
\text { Domain }\end{array}$ & 6 \\
\hline Hjermstad MJ [61] & 2005 & Cohort & $\begin{array}{l}\text { Cross- } \\
\text { sectional }\end{array}$ & $\begin{array}{l}\text { Median } 16.3 \text { years } \\
\text { since diagnosis }\end{array}$ & 496 & 46 median & Off & $\mathrm{FQ}$ & Symptom & 6 \\
\hline Ng AK [34] & 2005 & $\begin{array}{l}\text { Comparator: } \\
\text { Cohort }\end{array}$ & $\begin{array}{l}\text { Cross- } \\
\text { sectional }\end{array}$ & $\begin{array}{l}\text { Median } 15 \text { years } \\
\text { since diagnosis }\end{array}$ & 511 & 44 median & Off & FACIT-Fatigue & Symptom & 6 \\
\hline Wettergren L [62] & 2005 & Cohort & $\begin{array}{l}\text { Cross- } \\
\text { sectional }\end{array}$ & $\begin{array}{l}\text { Median } 14 \text { years } \\
\text { since diagnosis }\end{array}$ & 121 & NS & Off & SF-12, SEIQOL-DW & MultiD & 2 \\
\hline Hjermstad MJ [63] & 2006 & Cohort & $\begin{array}{l}\text { Cross- } \\
\text { sectional }\end{array}$ & $\begin{array}{l}\text { Median } 16.3 \text { years } \\
\text { since diagnosis }\end{array}$ & 479 & 46 mean & Off & SF-36, FQ & $\begin{array}{l}\text { MultiD, } \\
\text { Symptom }\end{array}$ & 5 \\
\hline Mols F [21] & 2006 & Cohort & $\begin{array}{l}\text { Cross- } \\
\text { sectional }\end{array}$ & $\begin{array}{l}5-15 \text { years since } \\
\text { diagnosis }\end{array}$ & 132 & NS & Off & SF-36, QoL-CS & MultiD & 6 \\
\hline Absolom K [64] & 2007 & Cohort & $\begin{array}{l}\text { Cross- } \\
\text { sectional }\end{array}$ & $\begin{array}{l}15.7 \text { years mean } \\
\text { since diagnosis }\end{array}$ & 50 & 39.7 mean & Off & SF-12, HADS & $\begin{array}{l}\text { MultiD, } \\
\text { Domain }\end{array}$ & 5 \\
\hline Aksnes LH [65] & 2007 & $\begin{array}{l}\text { Comparator: } \\
\text { Cohort }\end{array}$ & $\begin{array}{l}\text { Cross- } \\
\text { sectional }\end{array}$ & & 89 & 35 mean & Off & SF-36, HADS, FQ & & 4 \\
\hline
\end{tabular}


Table 2 Studies assessing HRQL in Hodgkin lymphoma patients $(N=65)$ (Continued)

\begin{tabular}{|c|c|c|c|c|c|c|c|c|c|c|}
\hline & & & & $\begin{array}{l}\text { Mean since diagnosis: } \\
14 \text { years for males, } \\
11 \text { years for females }\end{array}$ & & & & & $\begin{array}{l}\text { MultiD, } \\
\text { Domain, } \\
\text { Symptom }\end{array}$ & \\
\hline Oldervoll LM [66] & 2007 & Cohort & $\begin{array}{l}\text { Cross- } \\
\text { sectional }\end{array}$ & $\begin{array}{l}\text { Mean } 17 \text { years } \\
\text { since diagnosis }\end{array}$ & 476 & 46 mean & Off & $\mathrm{FQ}$ & Symptom & 5 \\
\hline Goodman KA [19] & 2008 & Cohort & $\begin{array}{l}\text { Cross- } \\
\text { sectional }\end{array}$ & $\begin{array}{l}\text { Median } 12 \text { years } \\
\text { post treatment }\end{array}$ & 60 & 43 median & Off & EORTC QLQ-C30 & MultiD & 6 \\
\hline Mulrooney DA [67] & 2008 & $\begin{array}{l}\text { Comparator: } \\
\text { Cohort }\end{array}$ & $\begin{array}{l}\text { Cross- } \\
\text { sectional }\end{array}$ & $\begin{array}{l}\text { At least } 15 \text { years } \\
\text { since diagnosis }\end{array}$ & 995 & NS & Off & $\begin{array}{l}\text { FACIT-Fatigue, Pittsburgh } \\
\text { Slee Quality Index, Epworth } \\
\text { Sleepiness Scale }\end{array}$ & Symptoms & 4 \\
\hline Shimoda S [68] & 2008 & Cohort & $\begin{array}{l}\text { Cross- } \\
\text { sectional }\end{array}$ & $\begin{array}{l}\text { Mean } 16.5 \text { years } \\
\text { since diagnosis }\end{array}$ & 15 & NS & Off & HUI3 & MultiD & 2 \\
\hline Kiserud CE [14] & 2009 & Cohort & $\begin{array}{l}\text { Cross- }^{-} \\
\text {sectional }\end{array}$ & $\begin{array}{l}\text { Mean } 15.2 \text { follow } \\
\text { up years }\end{array}$ & 138 & 45.7 mean & Off & $\begin{array}{l}\text { BSFI, Fatigue questionnaire, } \\
\text { HADS, SF-36 }\end{array}$ & $\begin{array}{l}\text { MultiD, } \\
\text { Symptom, } \\
\text { Domain }\end{array}$ & 6 \\
\hline Heutte N [1 1] & 2009 & $\begin{array}{l}\text { Comparator: } \\
\text { RCT }\end{array}$ & Longitudinal & $\begin{array}{l}\text { Mean } 7.5 \text { years } \\
\text { since diagnosis }\end{array}$ & 935 & 31 median & Off & $\begin{array}{l}\text { EORTC QLQ-C30, MFI, Sexual } \\
\text { Function Scale }\end{array}$ & $\begin{array}{l}\text { MultiD, } \\
\text { Symptoms }\end{array}$ & 6 \\
\hline Brandt J [69] & 2010 & Cohort & $\begin{array}{l}\text { Cross- } \\
\text { sectional }\end{array}$ & $\begin{array}{l}\text { Median since } \\
\text { diagnosis: HDCT } \\
11 \text { years, conventional } \\
\text { chemotherapy } \\
3.5 \text { years }\end{array}$ & 98 & $\begin{array}{l}46 \mathrm{HDCT}, 41 \\
\text { conventional } \\
\text { median }\end{array}$ & Off & EORTC QLQ-C30, EQ-5D & MultiD & 6 \\
\hline Klaassen RJ [40] & 2010 & Cohort & Longitudinal & $\begin{array}{l}2 \text { weeks after } \\
\text { first course of } \\
\text { chemotherapy }\end{array}$ & 49 & 14.7 mean & Both & $\begin{array}{l}\text { PedsQL, HUI2, HUI3, EuroQol, } \\
\text { Lanksy Play-Performance Score }\end{array}$ & $\begin{array}{l}\text { MultiD, } \\
\text { Other }\end{array}$ & 4 \\
\hline Klaassen RJ [41] & 2010 & $\begin{array}{l}\text { Comparator: } \\
\text { Cohort }\end{array}$ & Longitudinal & $\begin{array}{l}2 \text { weeks after } \\
\text { first course of } \\
\text { chemotherapy }\end{array}$ & 49 & 14.7 mean & Both & $\begin{array}{l}\text { PedsQL, HUI2, HUI3, EuroQol, } \\
\text { Lanksy Play-Performance Score }\end{array}$ & $\begin{array}{l}\text { MultiD, } \\
\text { Other }\end{array}$ & 2 \\
\hline Miltényi Z [17] & 2010 & Cohort & $\begin{array}{l}\text { Cross- } \\
\text { sectional }\end{array}$ & $\begin{array}{l}\text { Mean } 9.8 \text { years } \\
\text { since diagnosis }\end{array}$ & 168 & 43.11 median & Off & EORTC QLQ-C30 & MultiD & 6 \\
\hline Baptista RLR [70] & 2012 & Cohort & $\begin{array}{l}\text { Cross- }^{-} \\
\text {sectional }\end{array}$ & $\begin{array}{l}\text { Median } 7 \text { years } \\
\text { since diagnosis }\end{array}$ & 200 & 29 median & Off & $\mathrm{MFI}$ & Symptom & 4 \\
\hline Khimani N [18] & 2013 & Cohort & Longitudinal & $\begin{array}{l}\text { Median } 24 \text { years } \\
\text { since diagnosis }\end{array}$ & 273 & 52 median & Off & SF-36, FACIT-Fatigue & $\begin{array}{l}\text { MultiD, } \\
\text { Symptom }\end{array}$ & 5 \\
\hline Minn AY [20] & 2012 & Cohort & $\begin{array}{l}\text { Cross- } \\
\text { sectional }\end{array}$ & $\begin{array}{l}\text { Median } 10.2 \text { years } \\
\text { post treatment }\end{array}$ & 71 & 26 median & Off & EORTC QLQ-C30 & MultiD & 5 \\
\hline Behringer K [12] & 2013 & $\begin{array}{l}\text { Comparator: } \\
\text { RCT }\end{array}$ & Longitudinal & At diagnosis & 3208 & 36.4 mean & Both & $\begin{array}{l}\text { EORTC QLQ-C30, MFI, Sexual } \\
\text { Health Scale }\end{array}$ & $\begin{array}{l}\text { MultiD, } \\
\text { Symptoms }\end{array}$ & 6 \\
\hline Greaves P [39] & 2014 & $\begin{array}{l}\text { Comparator: } \\
\text { Cohort }\end{array}$ & $\begin{array}{l}\text { Cross- } \\
\text { sectional }\end{array}$ & $\begin{array}{l}\text { Mean } 20.3 \text { years } \\
\text { since diagnosis }\end{array}$ & 280 & 53.1 mean & Off & $\begin{array}{l}\text { FACT-BMT, IOC, Study-specific } \\
\text { questionnaire }\end{array}$ & $\begin{array}{l}\text { MultiD, } \\
\text { Other }\end{array}$ & 5 \\
\hline Hamre H [44] & 2013 & $\begin{array}{l}\text { Comparator: } \\
\text { Cohort }\end{array}$ & $\begin{array}{l}\text { Cross- } \\
\text { sectional }\end{array}$ & $\begin{array}{l}\text { Median } 21.5 \text { years } \\
\text { since diagnosis }\end{array}$ & 68 & 35 median & Off & $\mathrm{FQ}$ & Symptom & 5 \\
\hline
\end{tabular}


Table 2 Studies assessing HRQL in Hodgkin lymphoma patients $(N=65)$ (Continued)

\begin{tabular}{|c|c|c|c|c|c|c|c|c|c|c|}
\hline Kim S [71] & 2014 & Cohort & $\begin{array}{l}\text { Cross- } \\
\text { sectional }\end{array}$ & $\begin{array}{l}\text { Mean } 6.7 \text { years } \\
\text { since diagnosis }\end{array}$ & 58 & 43.3 mean & Off & EORTC QLQ-C30, HADS & $\begin{array}{l}\text { MultiD, } \\
\text { Domain }\end{array}$ & 6 \\
\hline Roper K [72] & 2013 & Cohort & Longitudinal & $\begin{array}{l}\text { At completion of } \\
\text { planned therapy }\end{array}$ & 40 & 30.9 mean & Off & SF-12, HADS, SDS, IOC & $\begin{array}{l}\text { MultiD, } \\
\text { Domain, } \\
\text { Other }\end{array}$ & 4 \\
\hline Soares A [45] & 2013 & Cohort & $\begin{array}{l}\text { Cross- } \\
\text { sectional }\end{array}$ & $\begin{array}{l}\text { Median } 7 \text { years } \\
\text { since diagnosis }\end{array}$ & 200 & 29 median & Off & SF-12, QOL-CS, MOS-SSS, MFI & $\begin{array}{l}\text { MultiD, } \\
\text { Symptom }\end{array}$ & 4 \\
\hline Vissers PAJ [15] & 2013 & Cohort & $\begin{array}{l}\text { Cross- } \\
\text { sectional }\end{array}$ & $\begin{array}{l}\text { Mean } 5 \text { years } \\
\text { since diagnosis }\end{array}$ & 150 & 47 mean & Off & EORTC QLQ-C30 & MultiD & 6 \\
\hline Calaminus G [46] & 2014 & Cohort & $\begin{array}{l}\text { Cross- } \\
\text { sectional }\end{array}$ & $\begin{array}{l}\text { Mean } 15.3 \text { years } \\
\text { since diagnosis }\end{array}$ & 725 & 28.44 mean & Off & EORTC QLQ-C30 & MultiD & 6 \\
\hline Daniels LA [31] & 2014 & Cohort & $\begin{array}{l}\text { Cross- } \\
\text { sectional }\end{array}$ & $\begin{array}{l}\text { Mean } 4.6 \text { years } \\
\text { since diagnosis }\end{array}$ & 180 & 46 mean & Off & $\begin{array}{l}\text { EORTC QLQ-C30, Fatigue Assessment } \\
\text { Scale, Study-specific questionnaire }\end{array}$ & $\begin{array}{l}\text { MultiD, } \\
\text { Symtom, } \\
\text { Domain }\end{array}$ & 6 \\
\hline Daniels LA [73] & 2014 & Cohort & Longitudinal & $\begin{array}{l}\text { Mean } 21 \text { years } \\
\text { since diagnosis }\end{array}$ & 43 & 47 mean & Off & $\begin{array}{l}\text { EORTC QLQ-C30, Fatigue Assessment } \\
\text { Scale, HADS }\end{array}$ & $\begin{array}{l}\text { MultiD, } \\
\text { Domain, } \\
\text { Symptom }\end{array}$ & 4 \\
\hline Oerlemans S [25] & 2014 & $\begin{array}{l}\text { Comparator: } \\
\text { Case Control }\end{array}$ & Longitudinal & $\begin{array}{l}\text { Mean } 4.7 \text { years } \\
\text { since diagnosis }\end{array}$ & 180 & 46.1 mean & Off & EORTC QLQ-C30, HADS & $\begin{array}{l}\text { MultiD, } \\
\text { Domain }\end{array}$ & 6 \\
\hline Vermaete N [47] & 2014 & $\begin{array}{l}\text { Comparator: } \\
\text { Cohort }\end{array}$ & Longitudinal & $\begin{array}{l}\text { Before the start } \\
\text { of chemotherapy }\end{array}$ & 12 & 47 mean & Both & EORTC QLQ-C30, Distress Barometer & $\begin{array}{l}\text { MultiD, } \\
\text { Domain }\end{array}$ & 5 \\
\hline Kiserud CE [74] & 2015 & Cohort & $\begin{array}{l}\text { Cross- } \\
\text { sectional }\end{array}$ & $\begin{array}{l}\text { Median } 16 \text { years } \\
\text { since diagnosis }\end{array}$ & 131 & 46 median & Off & Fatigue questionnaire, HADS, SF36, & $\begin{array}{l}\text { Symptom, } \\
\text { MultiD }\end{array}$ & 5 \\
\hline Husson O [42] & 2015 & $\begin{array}{l}\text { Comparator: } \\
\text { Cohort }\end{array}$ & $\begin{array}{l}\text { Cross- } \\
\text { sectional }\end{array}$ & $\begin{array}{l}\text { Mean } 5.3 \text { years } \\
\text { since diagnosis }\end{array}$ & 150 & 46.6 mean & Off & Fatigue assessment scale & Symptom & 4 \\
\hline
\end{tabular}

Abbreviations: Tx treatment, MultiD multidimensional, HL Hodgkin's Lymphoma, RCT randomized controlled trial, EORTC QLQ-C30 European Organisation for Research and Treatment of Cancer Quality of Life 3 . Questionnaire, SF-36/SF-12 Short Form, HUI Health Utilities Index, QOL-CS Quality of Life-Cancer Survivors, CES-D Center for Epidemiologic Studies Depression Scale, GSSI Global Sexual Satisfaction Index, IES Impact of Event Scale, VAS Visual Analogue Scale, CARES-SF Cancer

Rehabilitation Evaluation System-Short Form, SDS Symptom Distress Scale, SEIQoI-DW Schedule for the Evaluation of the Individual Quality of Life-Direct Weighting, PedsQL Pediatric Quality of Life Inventory, MOS-SSS Medical Outcomes Study-Social Support Survey, FACT-BMT Functional Assessment of Cancer Therapy-Bone Marrow Transplant, FQ Fatigue Questionnaire, MFI Multi-Dimensional Fatigue Inventory, FACIT-Fatigue Functional Assessment of Chronic Illness Therapy-Fatigue, HADS Hospital Anxiety and Depression Scale, BSI Brief Symptom Inventory, POMS Profile of Mood States, PAIS Psychosocial Adjustment to Illness Scale, HDCT high dose chemotherapy, STLI subtotal lymphoid irradiation, CMT combined modality treatment, NS not stated 
Table 3 Measure type by study design $(N=65)$

\begin{tabular}{lll}
\hline $\begin{array}{l}\text { HRQL measures, } \\
n(\%)^{a}\end{array}$ & $\begin{array}{l}\text { Longitudinal } \\
n=12(18)\end{array}$ & $\begin{array}{l}\text { Cross-sectional } \\
n=53(82)\end{array}$ \\
\hline $\begin{array}{l}\text { Multidimensional } \\
\text { Domain }\end{array}$ & $12(100)$ & $33(62)$ \\
$\quad$ Psychosocial & $4(33)$ & $20(38)$ \\
Sexual functioning & $2(17)$ & $5(9)$ \\
Physical & $1(8)$ & $0(0)$ \\
Symptom & \\
Fatigue & $4(33)$ & $18(34)$ \\
Pain & $0(0)$ & $1(2)$ \\
Other & $2(4)$ & $4(8)$ \\
\hline
\end{tabular}

a Percentages do not sum to 100 because multiple measures can be used in the same study, HRQL health-related quality of life

One longitudinal study with a small sample size showed that an exercise intervention can improve fatigue in $\mathrm{HL}$ survivors [36].

In our review, 63 (97\%) studies used at least one validated instrument. Two exceptions reported by Abrahamsen [29] and Chao [37], used only non-validated, study-specific questionnaires. Six other studies incorporated a study specific questionnaire in addition to a validated instrument. Of these, three included broad questions including demographic, medical and psychosocial information [26, 38, 39], while the other three created assessment tools specific to their study question pertaining to cardiac health [16], patient screening [31], and psychiatric disorders [32].

\section{Quality assessment}

Our quality assessment was based upon six criteria (Table 4$)$. Nearly $86 \%(n=56)$ of all of the studies included more than two demographic variables and clinical characteristics. The majority $(n=53,82 \%)$ also reported quality of life scores-specifically including a mean score and standard deviation (SD) in table or text

Table 4 Quality Assessment of Hodgkin Lymphoma Specific Studies

\begin{tabular}{lccc}
\hline Quality indicator & $\begin{array}{l}\text { Longitudinal } \\
(n=12)\end{array}$ & $\begin{array}{l}\text { Cross-sectional } \\
(n=53)\end{array}$ & $\begin{array}{l}\text { Total } \\
(N=65)\end{array}$ \\
\hline Sample >50 (HL Specific) & $6(42)$ & $45(85)$ & $51(78)$ \\
$\begin{array}{l}\text { Description of >=2 Demos } \\
\text { (HL Specific) }\end{array}$ & $10(83)$ & $48(91)$ & $58(89)$ \\
$\begin{array}{l}\text { Description of >=2 Clinical } \\
\text { Variables (HL Specific) }\end{array}$ & $11(92)$ & $45(85)$ & $56(86)$ \\
$\begin{array}{l}\text { HL Specific HRQL Scores } \\
\text { (from validated measure) }\end{array}$ & $8(67)$ & $45(85)$ & $53(82)$ \\
$\begin{array}{l}\text { Analysis of HL HRQL Scores } \times \\
\text { HL Characteristics }\end{array}$ & $8(67)$ & $37(70)$ & $45(69)$ \\
$\begin{array}{l}\text { HL Results are compared } \\
\text { between groups? }\end{array}$ & $9(75)$ & $44(83)$ & $53(82)$ \\
\hline
\end{tabular}

HL Hodgkin's Lymphoma, HRQL health-related quality of life form. Of the 11 studies that did not fulfill this criteria, five presented the data graphically [10, 12, 40-42]; three used the data to dichotomize groups (e.g., fatigued vs. non-fatigued population) [36, 43, 44]; two provided means scores but no standard deviation [41, 45]; one provided the percentages of negative impact of sexual function used to compare between groups [39], and one reported the percentage of sexual problems [29]. Most $(n=53,82 \%)$ of the studies compared results of individuals with HL with other groups such as siblings, general population or other individuals with cancer and $78 \%$ of the studies included a sample size greater than 50 . For the final criteria, $69 \%$ of the studies included an analysis of HL HRQL scores stratified by HL specific characteristics.

\section{Key articles}

The following section highlights specific studies with a focus on their methodological rigor. The 2013 prospective study by Behringer et al. [12] was embedded within German Hodgkin Study Group HD10-HD12 trials. This study included the largest sample within our review, 3208 patients, and followed these patients from diagnosis through up to 27 months of follow-up care. Patients were randomized to varying protocols of HL chemotherapy and radiation, according to each of the three clinical trials. This study ultimately focused on the sexual functioning (SX) domain; however it also collected multidimensional HRQL data. Studies instruments included the European Organization for Research and the Treatment of Cancer (EORTC-QLQ-C30), Multi-dimensional Fatigue Inventory (MFI) and a Sexual Functioning Scale. Researchers found that while SX was reduced at baseline, it improved after therapy and eventually normalized in individuals with early stage disease. Within the HD11 trial, which was a randomized comparison of doxorubicin, bleomycin, vinblastine, and dacarbazine (ABVD) versus bleomycin, etoposide, doxorubicin, cyclophosphamide, vincristine, procarbazine, and prednisone (BEACOPP), a small but significant difference of SX symptoms was detected in favor of ABVD. Long-term SX was related more to baseline SX and patient characteristics than to the intensity of treatment. Of note, older age, advanced stage disease, and female gender had an overall negative influence on SX.

From a quality perspective, the researchers used validated, multi-dimensional assessment tools; however they did not report specific means scores or the standard deviation. Instead, they used the HRQL data as independent variables to predict/explain sexual function and reported it in graph format. This absence of summary scores and variance precludes performing meta-analysis from these published data.

In 2003 Ganz et al. [10] conducted the only RCT of HRQL in HL in the US. This study was embedded in a RCT in cooperative Southwest Oncology Group 9133 
trial. It included 247 patients in a prospective, longitudinal design, evaluating multidimensional HRQL at baseline, 6 months, 1 and 2 years. Researchers found a statistical difference in HRQL between the two treatments subtotal lymphoid irradiation with combined modality treatment (CMT). Specifically, the CMT arm experienced greater symptom distress, fatigue and poorer overall HRQL; however, by years 1 to 2, patients with in the two groups did not differ in outcomes. The authors also reported that both groups experienced significantly increased fatigue at 6 months from baseline (when compared to healthy populations) and this persisted even 1-2 years after treatment.

This study used a high quality methodology with a prospective, longitudinal, RCT design, a large sample size, and validated multi-dimensional assessment tools; however, like Behringer [12], they did not report specific means scores or the standard deviation, but instead presented trends over time graphically.

Heutte et al. [11] conducted a prospective, longitudinal study assessing HRQL among patients treated on the H8 study in Europe. This study included 935 patients, who were assessed at the end of therapy and serially up 10 years following treatment using the EORTC-QLQ-C30, MFI, and sexual function scale. Their results indicated that the emotional functioning scores were more affected than the physical functioning scores, and that women reported lower HRQL and increased symptom distress than men. All of the HRQL domains they evaluated showed improvement within 18 months of treatment completion with the exception of cognitive function and motivation. The authors suggest that neither of these domains were affected by the HL or treatment. Finally, they found that high levels of fatigue at end of treatment predicted persistent fatigue into longterma follow-up. They did not find any differences between the treatment groups. Methodologically, this study included a large xsample size, randomization between treatment groups and used validated, multi-dimensional assessment tools. Methodologic concerns included the lack of baseline data (prior to initiation of treatment) and no data obtained during treatment.

Calaminus et al. [46] conducted a cross-sectional study assessing HRQL in pediatric survivors, who were previously treated for HL in German-Austrian RCT studies from 1978 to 2002. This study enrolled 725 participants, who were assessed using the EORTC QLQ-C30. Results were compared to a similarly matched general population sample. Several of the results of this study are similar to Heutte's: survivors experienced worse emotional and social functioning compared to normal population; females experience lower over-all functioning and higher symptom burden than men; survivors experienced greater symptoms of fatigue and sleep problems. Again, there was no relationship between the treatment types. Methodologically, this study included a large sample size, validated instruments and comparisons between groups. Although it did not follow patients over time, the study does illustrate how a well-designed cross sectional study can support longitudinal findings.

Klaassen et al. [40] examined the ability to detect change over time in four different HRQL measures. In their prospective study of 48 patients, patients were assessed at four points in time: 2 weeks after first course of chemotherapy, during the second course of chemotherapy, during their week of radiation and 1 year after diagnosis. All of the HRQL measures demonstrated significant change from Time 1 to Time 4. In a second study, Klaassen [41] also examined proxy reporting by parents and nurses of children with HL to determine if correlation with children's report. In this study, the authors found that over the course of treatment there was statistical significant agreement among the child, parent and nurse, as measured by the Spearman rank-order correlation coefficient. Methodologically, this study used validated tools and compared trends over time; however, concerns include its small sample size, no baseline data, no randomization according to the treatment arm or disease stage and the absence of means scores with standard deviations.

Vermaete et al. [47] conducted a longitudinal study to assess fatigue, physical activity and physical fitness in individuals with lymphoma before, during and after treatment. This study included 29 patients with either HL $(n=12)$ or Non-Hodgkin Lymphoma $(n=17)$. Over course of treatment, researchers detected a decline in hemoglobin, physical force and oxygen uptake, and patients reported significantly increased fatigue. This study looks deeper into the complex relationship between fatigue, physical inactivity and deconditioning and support further work in developing exercise interventions. Methodologically, this study used validated tools, obtained baseline prior to the start of treatment and provided mean scores with standard deviation; however, it enrolled a small sample size and did not randomize according to the treatment arm or disease stage.

\section{Discussion}

In our review the majority of studies employed crosssectional versus longitudinal design and of these crosssectional studies, $98 \%$ enrolled participants off treatment. These cross sectional studies capture patients within a wide range of time periods after diagnosis, on average, 10 years after treatment. While cross sectional studies provide a "snap shot in time," analyzing a group one decade after treatment introduces the risk of many confounding variables, which makes it difficult to build a cohesive narrative around the impact of $\mathrm{HL}$ and its treatment on HRQL. In terms of assessing HRQL, all longitudinal studies used a multi-dimensional measure compared to $62 \%$ of 
cross-sectional studies, which focused more on specific domains and symptoms, especially fatigue.

The objective of this review was to systematically identify published studies reporting HRQL in individuals with HL, and to examine the quality of these studies. Our analysis points to several positive trends in the literature. First, it is clear that that over time there has been a growing interest in this topic, as the number of studies that examine HRQL has increased. As noted, half of the studies identified in this review were published after $2005(n=34)$. The second positive trend is movement toward more longitudinal studies with nine of the 12 published since 2009. In regard to patient reported outcomes in HRQL, the majority of studies used at least one multidimensional instrument $(n=45)$ and $97 \%$ of studies included at least one validated instrument.

We also noted several concerns with quality of the current literature. The most rigorous methodology employs a longitudinal design with comparison between groups and changes within subjects. Our review identified only 12 longitudinal studies with ten unique data sets. Only five out of the 12 studies followed patients from diagnosis through to post-treatment and of these only two included at least 50 patients. Reporting HRQL data also varied across the longitudinal studies with four reporting results graphically without mean summary scores and measures of variability [10, 12, 40, 41], which prevents further meta-analysis.

Behringer and colleagues [12] illustrate the complex and informative data that can be derived from prospective, longitudinal designs. It provides clinician's with a deeper understanding of the trajectory of SX from diagnosis through treatment; additionally, it describes how SX differs across treatment groups and identifies those specific populations, who are most vulnerable. This information can assist clinicians in providing anticipatory guidance and targeted-interventions to the most vulnerable populations.

Results from Ganz and colleagues [10] inform clinicians that symptom burden can vary between treatment groups; however fatigue can persist over time. Clinicians could use this information to guide CMT patients to be more realistic with short-term goals immediately after treatment and to initiate more aggressive symptom management interventions. Second, in regard to fatigue, clinicians could use this information to prepare their patients for fatigue, continue to assess for fatigue during follow-up care and to support further research and interventions in fatigue prevention and management.

\section{Relevance to research and clinical practice}

This systematic review reveals the paucity of information on the HRQL impact of initial diagnosis and treatment on HL patients. The majority of published studies are cross-sectional in design and relatively small in size; only 36 studies have sample sizes $>100$ (Table 2). This limits the application of study findings due to concerns about generalizability and reproducibility. That said, the few longitudinal studies commencing prior to the initiation of treatment, detailed on page 11, provide such information about HRQL trajectory and how it changes over time and by treatment. These findings offer compelling evidence for the need to replicate these measures in future trials.

This study has several strengths. First, we followed PRISMA guidelines to systematically search the literature to capture the complete and relevant published literature. Second, the quality assessment methods provided a standardized measure by which all articles were evaluated. Last, we highlighted the subset of articles which exemplify best practices to examine HRQL in HL patients, with the goal of building upon these methods and findings in future research.

\section{Limitations}

There are limitations to this study. First, because of the lack of RCTs on this topic, we included observational studies, which are more open to bias. Second, we did not conduct an assessment of publication bias as our aim was to analyze the quality rather than to metaanalyze the findings. Last, most HRQL assessments were completed post-treatment so the longitudinal trajectory of HRQL was not captured. More large-cohort, prospective studies are needed to address this limitation.

\section{Conclusions}

$\mathrm{HL}$ is a highly curable disease with standardized treatment paradigms over the last two decades. Although the treatment is well established, a knowledge gap still exists in understanding how this diagnosis and its treatment affect the individual's HRQL from diagnosis though long-term survivorship. Further, with the exception of an ongoing pediatric cooperative group trial, we found no prospective pediatric studies reporting on HRQL in HL from diagnosis to survivorship. Even in adult studies, we identified a substantial void of HRQL data during the active treatment phase. Finally, we identified no industry-funded studies, although this may change with the emergence of novel therapeutics.

With the growing interest and acknowledgement of the importance of HRQL, we recommend that future research studies employ greater methodological rigor by including prospective, longitudinal randomized designs across both treatment and time. Behringer [12], Heutte et al. [11] and Ganz [10], provide a "gold standard" of research studies that not only examine longitudinal effects within subject changes, but also provide comparisons between different treatment regimens. The information 
generated by these longitudinal studies helps clinicians target vulnerable populations and provide anticipatory guidance to patients. Such studies will generate further data that clinicians can use to address "real life" HRQL problems that patients face on a day-to-day basis. Further, while research supports that HRQL improves after treatment, continued deficits in some patients within the domains of fatigue, sexual and psychosocial health warrants further study with targeted interventions to mitigate the risk of poorer HRQL. Finally, as the focus on HRQL continues to grow in importance, researchers should consider partnering with industry to examine oncologic treatments within the context of how they will impact the patient's HRQL.

\begin{abstract}
Abbreviations
BSI brief symptom inventory; CARES-SF cancer rehabilitation evaluation system-short form; CES-D center for epidemiologic studies depression scale; CMT combined modality treatment; EORTC QLQ-C30 European Organisation for Research and Treatment of Cancer Quality of Life Questionnaire; FACIT-Fatigue functional assessment of chronic illness therapy-fatigue; FACT-BMT functional assessment of cancer therapy—-bone marrow transplant; $F Q$ fatigue questionnaire; GSSI global sexual satisfaction index; HADS Hospital anxiety and depression scale; HDCT high dose chemotherapy; HL Hodgkin's lymphoma; HRQL health-related quality of life; HUI health utilities index; IES impact of event scale; MFI multi-dimensional fatigue inventory; MOS-SSS medical outcomes study-social support survey; MultiD multidimensional; NS not stated; PAIS psychosocial adjustment to illness scale; PedsQL pediatric quality of life inventory; POMS profile of mood states; QA quality assessment; QOL-CS quality of life-cancer survivors; RCT randomized controlled trial; SD standard deviation; SDS symptom distress scale; SEIQol-DW schedule for the evaluation of the individual quality of life-direct weighting; SF-36/SF-12 short form; STLI subtotal lymphoid irradiation; SX sexual functioning; $T X$ treatment; VAS visual analogue scale
\end{abstract}

\section{Acknowledgements}

The authors would like to thank Darcy Banco and Katherine Spencer for their contributions and the Sacco family for their generous support.

\section{Funding}

This study was funded in full by the Reid R. Sacco Adolescent and Young Adult Cancer Alliance. The funder had no role in the study design, data collection, analysis, and interpretation or writing of the manuscript.

\section{Authors' contributions}

$\mathrm{NL}, \mathrm{TS}$, and SP contributed to study design, data acquisition, data analysis, manuscript preparation, and final approval. RB, JN, KW and AE contributed to the study design, data acquisition, manuscript preparation, and final approval. All authors agree to be accountable for the content of the manuscript as submitted.

\section{Competing interests}

Author Andrew M. Evens, DO, MSc, has received honoraria from the following companies: Millennium: research and advisory board with honorarium, Seattle Genetics: advisory board with honorarium, and Celgene: advisory board with honorarium. No other authors have any potential conflicts of interest.

\section{Consent for publication}

Not applicable.

\section{Ethics approval and consent to participate}

Not applicable. This article does not contain any studies with human participants performed by any of the authors and this article does not contain any studies with animals performed by any of the authors.

\section{Author details}

'Tufts Cancer Center, Tufts Medical Center, 800 Washington Street, \#245, Boston, MA 02111, USA. ${ }^{2}$ Institute for Clinical Research and Health Policy, Tufts Medical Center, 800 Washington Street, \#345, Boston, MA 02111, USA. ${ }^{3}$ Advocate Lutheran General Hospital, 1775 Dempster St, Park Ridge, IL 60068, USA. ${ }^{4}$ Tufts University School of Medicine, 145 Harrison Avenue, Boston, MA 02111, USA.

Received: 20 February 2016 Accepted: 25 July 2016

Published online: 29 July 2016

\section{References}

1. DeVita Jr VT, Simon RM, Hubbard SM, Young RC, Berard CW, Moxley III JH, et al. Curability of advanced Hodgkin's disease with chemotherapy. Longterm follow-up of MOPP-treated patients at the National Cancer Institute. Ann Intern Med. 1980;92:587-95.

2. Canellos GP, Anderson JR, Propert KJ, Nissen N, Cooper MR, Henderson ES, et al. Chemotherapy of advanced Hodgkin's disease with MOPP, ABVD, or MOPP alternating with ABVD. N Engl J Med. 1992;327:1478-84.

3. Surveillance, Epidemiology, and End Results Program. SEER Stat Fact Sheets: Hodgkin Lymphoma. National Cancer Institute. http://seer.cancer.gov/ statfacts/html/hodg.html. Accessed 15 Sept 2015.

4. American Cancer Society. What are the Key Statistics about Hodgkin Disease? 2015. http://www.cancer.org/cancer/hodgkindisease/ detailedguide/hodgkin-disease-key-statistics. Accessed 15 Sept 2015.

5. International Health Conference. Constitution of the World Health Organization. 1946. Bull World Health Organ. 2002;80(12):983-4.

6. Croog SH, Levine S, Testa MA, Brown B, Bulpitt CJ, Jenkins CD, et al. The effects of antihypertensive therapy on the quality of life. N Engl J Med. 1986;314:1657-64

7. Moher D, Liberati A, Tetzlaff J, Altman DG. Preferred reporting items for systematic reviews and meta-analyses: the PRISMA statement. PLoS Med. 2009;6:e1000097.

8. Daniels LA, Oerlemans S, Krol AD, van de Poll-Franse LV, Creutzberg CL. Persisting fatigue in Hodgkin lymphoma survivors: a systematic review. Ann Hematol. 2013;92:1023-32.

9. Oerlemans S, Mols F, Nijziel MR, Lybeert M, van de Poll-Franse LV. The impact of treatment, socio-demographic and clinical characteristics on health-related quality of life among Hodgkin's and non-Hodgkin's lymphoma survivors: a systematic review. Ann Hematol. 2011;90:993-1004.

10. Ganz PA, Moinpour CM, Pauler DK, Kornblith AB, Gaynor ER, Balcerzak SP, et al. Health status and quality of life in patients with early-stage Hodgkin's disease treated on Southwest Oncology Group Study 9133. J Clin Oncol. 2003:21:3512-9.

11. Heutte N, Flechtner HH, Mounier N, Mellink WA, Meerwaldt JH, Eghbali H, et al. Quality of life after successful treatment of early-stage Hodgkin's lymphoma: 10-year follow-up of the EORTC-GELA H8 randomised controlled trial. Lancet Oncol. 2009:10:1160-70.

12. Behringer $\mathrm{K}$, Muller $\mathrm{H}$, Gorgen $\mathrm{H}$, Flechtner HH, Brillant C, Halbsguth TV, et al. Sexual quality of life in Hodgkin Lymphoma: a longitudinal analysis by the German Hodgkin Study Group. Br J Cancer. 2013;108:49-57.

13. Greil R, Holzner B, Kemmler G, Kopp M, Buchowski A, Oberaigner W, et al. Retrospective assessment of quality of life and treatment outcome in patients with Hodgkin's disease from 1969 to 1994. Eur J Cancer. 1999;35:698-706.

14. Kiserud CE, Schover LR, Dahl AA, Fossa A, Bjoro T, Loge JH, Holte H, Yuan Y, Fossa SD. Do male lymphoma survivors have impaired sexual function? J Clin Oncol. 2009;27:6019-26.

15. Vissers PA, Thong MS, Pouwer F, Zanders MM, Coebergh JW, van de PollFranse LV. The impact of comorbidity on Health-Related Quality of Life among cancer survivors: analyses of data from the PROFILES registry. J Cancer Surviv. 2013;7:602-13.

16. Adams MJ, Lipsitz SR, Colan SD, Tarbell NJ, Treves ST, Diller L, et al. Cardiovascular status in long-term survivors of Hodgkin's disease treated with chest radiotherapy. J Clin Oncol. 2004;22:3139-48.

17. Miltenyi Z, Magyari F, Simon Z, Illes A. Quality of life and fatigue in Hodgkin's lymphoma patients. Tumori. 2010;96:594-600.

18. Khimani N, Chen YH, Mauch PM, Recklitis C, Diller L, Silver B, et al. Influence of new late effects on quality of life over time in Hodgkin Iymphoma Survivors: a longitudinal survey study. Ann Oncol. 2013;24:226-30 
19. Goodman KA, Riedel E, Serrano V, Gulati S, Moskowitz CH, Yahalom J. Long-term effects of high-dose chemotherapy and radiation for relapsed and refractory Hodgkin's lymphoma. J Clin Oncol. 2008;26:5240-7.

20. Minn AY, Riedel E, Halpern J, Johnston LJ, Horning SJ, Hoppe RT, et al. Long-term outcomes after high dose therapy and autologous haematopoietic cell rescue for refractory/relapsed Hodgkin lymphoma. Brit J Haematol. 2012;159:329-39.

21. Mols F, Vingerhoets AJ, Coebergh JW, Vreugdenhil G, Aaronson NK, Lybeert ML, et al. Better quality of life among 10-15 year survivors of Hodgkin's lymphoma compared to 5-9 year survivors: a population-based study. Eur J Cancer. 2006:42:2794-801.

22. Kornblith AB, Anderson J, Cella DF, Tross S, Zuckerman E, Cherin E, et al. Quality of life assessment of Hodgkin's disease survivors: a model for cooperative clinical trials. Oncology (Williston Park). 1990;4:93-101.

23. Kornblith AB, Anderson J, Cella DF, Tross S, Zuckerman E, Cherin E, et al. Hodgkin disease survivors at increased risk for problems in psychosocial adaptation. The Cancer and Leukemia Group B. Cancer. 1992;70:2214-24.

24. Kornblith AB, Herndon JE, Zuckerman E, Cella DF, Cherin E, Wolchok S, et al. Comparison of psychosocial adaptation of advanced stage Hodgkin's disease and acute leukemia survivors. Cancer and Leukemia Group B. Ann Oncol. 1998;9:297-306.

25. Oerlemans S, Mols F, Nijziel MR, Zijlstra WP, Coebergh JW, van de PollFranse LV. The course of anxiety and depression for patients with Hodgkin's lymphoma or diffuse large B cell lymphoma: a longitudinal study of the PROFILES registry. J Cancer Surviv. 2014;8:555-64.

26. Fobair P, Hoppe RT, Bloom J, Cox R, Varghese A, Spiegel D. Psychosocial problems among survivors of Hodgkin's disease. J Clin Oncol. 1986:4:805-14.

27. Gil-Fernandez J, Ramos C, Tamayo T, Tomas F, Figuera A, Arranz R, et al. Quality of life and psychological well-being in Spanish long-term survivors of Hodgkin's disease: results of a controlled pilot study. Ann Hematol. 2003;82:14-8

28. Wettergren $L$, Bjorkholm M, Axdorph U, Langius-Eklof A. Determinants of health-related quality of life in long-term survivors of Hodgkin's lymphoma. Qual Life Res. 2004;13:1369-79.

29. Abrahamsen AF, Loge JH, Hannisdal E, Holte H, Kvaloy S. Socio-medical situation for long-term survivors of Hodgkin's disease: a survey of 459 patients treated at one institution. Eur J Cancer. 1998;34:1865-70.

30. van Tulder MW, Aaronson NK, Bruning PF. The quality of life of long-term survivors of Hodgkin's disease. Ann Oncol. 1994;5:153-8.

31. Daniels LA, Oerlemans S, Krol AD, Creutzberg CL, van de Poll-Franse LV. Chronic fatigue in Hodgkin lymphoma survivors and associations with anxiety, depression and comorbidity. Br J Cancer. 2014;110:868-74.

32. Loge $\mathrm{JH}$, Abrahamsen AF, Ekeberg, Kaasa S. Fatigue and psychiatric morbidity among Hodgkin's disease survivors. J Pain Symptom Manage. 2000;19:91-9.

33. Ruffer JU, Flechtner $H$, Tralls $P$, Josting $A$, Sieber $M$, Lathan $B$, et al. Fatigue in long-term survivors of Hodgkin's lymphoma; a report from the German Hodgkin Lymphoma Study Group (GHSG). Eur J Cancer. 2003;39:2179-86.

34. Ng AK, Li S, Recklitis C, Neuberg D, Chakrabarti S, Silver B, et al. A comparison between long-term survivors of Hodgkin's disease and their siblings on fatigue level and factors predicting for increased fatigue. Ann Oncol. 2005;16:1949-55.

35. Knobel H, Havard LJ, Lund MB, Forfang K, Nome O, Kaasa S. Late medical complications and fatigue in Hodgkin's disease survivors. J Clin Oncol. 2001:19:3226-33.

36. Oldervoll LM, Kaasa S, Knobel H, Loge JH. Exercise reduces fatigue in chronic fatigued Hodgkins disease survivors-results from a pilot study. Eur J Cancer. 2003;39:57-63.

37. Chao NJ, Tierney DK, Bloom JR, Long GD, Barr TA, Stallbaum BA, et al. Dynamic assessment of quality of life after autologous bone marrow transplantation. Blood. 1992:80:825-30.

38. Joly F, Henry-Amar M, Arveux P, Reman O, Tanguy A, Peny A, et al. Late psychosocial sequelae in hodgkin's disease survivors: a french populationbased case-control study. J Clin Oncol. 1996;14:2444-53.

39. Greaves P, Sarker SJ, Chowdhury K, Johnson R, Matthews J, Matthews R, et al. Fertility and sexual function in long-term survivors of haematological malignancy: using patient-reported outcome measures to assess a neglected area of need in the late effects clinic. Brit J Haematol. 2014;164:526-35.

40. Klaassen RJ, Krahn M, Gaboury I, Hughes J, Anderson R, Grundy P, et al. Evaluating the ability to detect change of health-related quality of life in children with Hodgkin disease. Cancer. 2010;116:1608-14.

41. Klaassen RJ, Barr RD, Hughes J, Rogers P, Anderson R, Grundy P, et al. Nurses provide valuable proxy assessment of the health-related quality of life of children with Hodgkin disease. Cancer. 2010;116:1602-7.

42. Husson O, Mols F, van de Poll-Franse L, de Vries J, Schep G, Thong MS. Variation in fatigue among 6011 (long-term) cancer survivors and a normative population: a study from the population-based PROFILES registry. Support Care Cancer. 2015;23:2165-74.

43. Zebrack BJ, Zeltzer LK, Whitton J, Mertens AC, Odom L, Berkow R, et al. Psychological outcomes in long-term survivors of childhood leukemia, Hodgkin's disease, and non-Hodgkin's lymphoma: a report from the Childhood Cancer Survivor Study. Pediatrics. 2002;110:42-52.

44. Hamre H, Zeller B, Kanellopoulos A, Ruud E, Fossa SD, Loge JH, et al. Serum cytokines and chronic fatigue in adults surviving after childhood leukemia and lymphoma. Brain Behav Immun. 2013;30:80-7.

45. Soares A, Biasoli I, Scheliga A, Baptista RL, Brabo EP, Morais JC, et al. Association of social network and social support with health-related quality of life and fatigue in long-term survivors of Hodgkin lymphoma. Support Care Cancer. 2013;21:2153-9.

46. Calaminus G, Dorffel W, Baust K, Teske C, Riepenhausen M, Bramswig $J$, et al. Quality of life in long-term survivors following treatment for Hodgkin's disease during childhood and adolescence in the German multicentre studies between 1978 and 2002. Support Care Cancer. 2014:22:1519-29.

47. Vermaete N, Wolter P, Verhoef G, Gosselink R. Physical activity and physical fitness in lymphoma patients before, during, and after chemotherapy: a prospective longitudinal study. Ann Hematol. 2014;93:411-24.

48. Kornblith AB, Anderson J, Cella DF, Tross S, Zuckerman E, Cherin E, et al. Comparison of psychosocial adaptation and sexual function of survivors of advanced Hodgkin disease treated by MOPP, ABVD, or MOPP alternating with ABVD. Cancer. 1992;70:2508-16.

49. Bloom JR, Fobair P, Gritz E, Wellisch D, Spiegel D, Varghese A, et al. Psychosocial outcomes of cancer: a comparative analysis of Hodgkin's disease and testicular cancer. J Clin Oncol. 1993;11:979-88.

50. Norum J, Wist E. Psychological distress in survivors of Hodgkin's disease. Support Care Cancer. 1996:4:191-5.

51. Norum J, Angelsen V, Wist E, Olsen JA. Treatment costs in Hodgkin's disease: a cost-utility analysis. Eur J Cancer. 1996;32A:1510-7.

52. Norum J, Wist EA. Quality of life in survivors of Hodgkin's disease. Qual Life Res. 1996;5:367-74.

53. Kaasa S, Loge JH, Knobel H, Jordhoy MS, Brenne E. Fatigue. Measures and relation to pain. Acta Anaesthesiol Scand. 1999;43:939-47.

54. Loge JH, Abrahamsen AF, Ekeberg O, Kaasa S. Hodgkin's disease survivors more fatigued than the general population. J Clin Oncol. 1999;17:253-61.

55. Loge JH, Abrahamsen AF, Ekeberg O, Kaasa S. Reduced health-related quality of life among Hodgkin's disease survivors: a comparative study with general population norms. Ann Oncol. 1999;10:71-7.

56. Van Schaik CS, Barr RD, Depauw S, Furlong W, Feeny D. Assessment of health status and health-related quality of life in survivors of Hodgkin's disease in childhood. Int J Cancer Suppl. 1999;12:32-8.

57. Barr RD, Gonzalez A, Longchong M, Furlong W, Vizcaino MP, Horsman $J$, et al. Health status and health-related quality of life in survivors of cancer in childhood in Latin America: a MISPHO feasibility study. Int J Oncol. 2001;19:413-21.

58. Cameron CL, Cella D, Herndon JE, Kornblith AB, Zuckerman E, Henderson E, et al. Persistent symptoms among survivors of Hodgkin's disease: an explanatory model based on classical conditioning. Health Psychol. 2001;20:71-5.

59. Zabora J, Brintzenhofeszoc K, Curbow B, Hooker C, Piantadosi S. The prevalence of psychological distress by cancer site. Psychooncology. 2001;10:19-28

60. Wettergren L, Bjorkholm M, Axdorph U, Bowling A, Langius-Eklof A. Individual quality of life in long-term survivors of Hodgkin's lymphoma - a comparative study. Qual Life Res. 2003;12:545-54.

61. Hjermstad MJ, Fossa SD, Oldervoll L, Holte H, Jacobsen AB, Loge JH. Fatigue in long-term Hodgkin's Disease survivors: a follow-up study. J Clin Oncol. 2005;23:6587-95. 
62. Wettergren L, Bjorkholm M, Langius-Eklof A. Validation of an extended version of the SEIQOL-DW in a cohort of Hodgkin lymphoma' survivors. Qual Life Res. 2005;14:2329-33.

63. Hjermstad MJ, Oldervoll L, Fossa SD, Holte H, Jacobsen AB, Loge JH. Quality of life in long-term Hodgkin's disease survivors with chronic fatigue. Eur J Cancer. 2006:42:327-33.

64. Absolom K, Greenfield D, Ross R, Davies H, Hancock B, Eiser C. Reassurance following breast screening recall for female survivors of Hodgkin's lymphoma. Breast. 2007;16:590-6.

65. Aksnes LH, Hall KS, Jebsen N, Fossa SD, Dahl AA. Young survivors of malignant bone tumours in the extremities: a comparative study of quality of life, fatigue and mental distress. Support Care Cancer. 2007;15:1087-96.

66. Oldervoll LM, Loge JH, Kaasa S, Lydersen S, Hjermstad MJ, Thorsen L, et al. Physical activity in Hodgkin's lymphoma survivors with and without chronic fatigue compared with the general population - a cross-sectional study. BMC Cancer. 2007;7:210.

67. Mulrooney DA, Ness KK, Neglia JP, Whitton JA, Green DM, Zeltzer LK, et al. Fatigue and sleep disturbance in adult survivors of childhood cancer: a report from the childhood cancer survivor study (CCSS). Sleep. 2008;31:271-81.

68. Shimoda S, Horsman J, Furlong W, Barr R, de Camargo B. Disability and health-related quality of life in long-term survivors of cancer in childhood in Brazil. J Pediatr Hematol Oncol. 2008;30:563-70.

69. Brandt J, Dietrich S, Meissner J, Neben K, Ho AD, Witzens-Harig M. Quality of life of long-term survivors with Hodgkin lymphoma after high-dose chemotherapy, autologous stem cell transplantation, and conventional chemotherapy. Leuk Lymphoma. 2010;51:2012-20.

70. Baptista RL, Biasoli I, Scheliga A, Soares A, Brabo E, Morais JC, et al. Psychometric properties of the multidimensional fatigue inventory in Brazilian Hodgkin's lymphoma survivors. J Pain Symptom Manage. 2012;44:908-15.

71. Kim SH, Kim IR, Kim SH, Lee S, Ok O, Kim WS, et al. Health-related quality of life in Korean lymphoma survivors compared with the general population. Ann Hematol. 2014;93:1531-40

72. Roper K, Cooley M, McDermott K, Fawcett J. Health-related quality of life after treatment of Hodgkin Lymphoma in Young adults. Oncol Nurs Forum. 2013;40:349-60.

73. Daniels LA, Krol SD, de Graaf MA, Scholte AJ, van't Veer MB, Putter $H$, et al. Impact of cardiovascular counseling and screening in Hodgkin lymphoma survivors. Int J Radiat Oncol Biol Phys. 2014;90:164-71.

74. Kiserud CE, Seland M, Holte H, Fossa A, Fossa SD, Bollerslev J, Bjoro T, Loge $\mathrm{JH}$. Fatigue in male lymphoma survivors differs between diagnostic groups and is associated with latent hypothyroidism. Acta Oncol. 2015;54:49-59.

\section{Submit your next manuscript to BioMed Central and we will help you at every step:}

- We accept pre-submission inquiries

- Our selector tool helps you to find the most relevant journal

- We provide round the clock customer support

- Convenient online submission

- Thorough peer review

- Inclusion in PubMed and all major indexing services

- Maximum visibility for your research

Submit your manuscript at www.biomedcentral.com/submit

) Biomed Central 